\title{
The Use of Cryotherapy in the Gout Pain Control: A Systematic Review
}

\author{
Alana Ludemila de Freitas Tavares, BSc; Iranilda Moha Ross, BSc ; Lilian Araujo Pradal, BSc; \\ Morgana Neves, MSc; Bárbara Heloise Trombetta Jamilk, BSc ; Gladson Ricardo Flor Bertolini, Ph.D \\ Western Paraná State University, Cascavel - Paraná, Brazil
}

Background Gout traditional treatment consists of anti-inflammatory drugs, analgesics and other rheumatic controllers, however, the occurrence of drug interactions occurs with a certain degree of toxicity, requiring that other methods be used to relieve symptoms. An alternative treatment is cryotherapy due to its capacity for analgesia.

Purpose The aim of this study was to conduct a systematic review that addressed the literature with production on gouty arthritis and its treatment with cryotherapy.

Study design Systematic review

Methods This review was described according to the items requested by the PRISMA system. This is a systematic review of publications from the year 2000 to the year 2019 in the following white literature databases PubMed, LILACS, SciELO, Web of Science, Science Direct, Scopus, Cochrane, PEDro and in the gray literature a Google Scholar database. The electronic databases were consulted from November 2019.

Results After searching the databases, reading and selection, two studies contemplated the methodological criteria of eligibility, being of the same authorship, both with more than ten years of publication, using similar methods of application of ice.

Conclusions It was found that, despite the scarcity of studies that addressed the topic, the articles found show reasonable rigor methodological and homogeneous results, demonstrating that cryotherapy helps in reducing the pain of individuals with gout, however, there is little evidence to support this hypothesis.

Key words Arthropathies; Cryotherapy; Gouty arthritis; Hypothermia; Ice; Pain relievers.

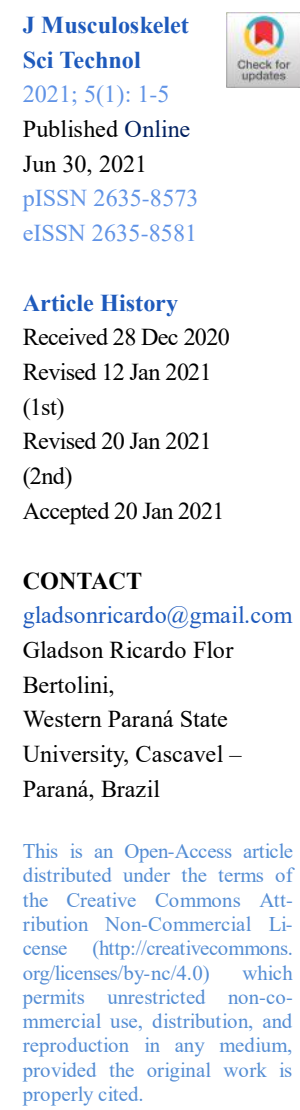

J Musculoskelet

DISSN 2635-8573

Article History

Received 28 Dec 2020

Revised 12 Jan 202

Revised 20 Jan 2021

(2nd)

\section{CONTACT}

Gladson Ricardo Flor

Bertolini,

Western Paraná State

University, Cascavel -

Paraná, Brazil

\section{INTRODUCTION}

Gouty arthritis is an acute inflammatory disease that affects intra and peri-articular tissues with single joint first, ${ }^{1}$ evolving into polyarticular with monoarticular onset, evolving to polyarticular resulting from hyperuricemia and deposition of crystals in the joint. ${ }^{2}$ It has a high prevalence in the male population, mainly from the age of 40 with the drop in hormonal rates. ${ }^{3,4}$

Uric acid, resulting from the purine metabolism, does not undergo oxidative degradation due to a deficiency of the liver enzyme uricase, thus it is the final product to be ex- creted, however, as it is less soluble, its serum level is close to its solubility, about $6.8 \mathrm{mg} / \mathrm{dL}$, factors that contribute to elevated serum levels include a diet rich in purines, hypoexcretion, age, sex, metabolic and renal diseases. ${ }^{5}$

Pathological levels of uric acid, triggers its crystallization and accumulation in the joints. These crystals are adjuvant in the beginning of the inflammatory process, stimulating the release of inflammatory mediators such as interleukins and tumor necrosis factors (TNF- $\alpha)^{6}$

Clinical signs of gouty arthritis occur as a result of this inflammatory response to crystals, ${ }^{7}$ with clinical picture showing local symptoms of acute arthritis, such as pain, 
hyperemia, hyperthermia, edema and loss of function, with peak pain of approximately 6 to 8 hours, usually sudden onset during the night. ${ }^{8,9}$

Traditional treatments have the function of reducing pain and inflammation caused by crystals. The use of medications is recommended, with non-steroidal anti-inflammatory drugs, colchicine and prednisone being the drugs of choice in the acute period of the disease. ${ }^{10}$ These drugs are efficient, however they have unpleasant side effects, intestinal and renal and are dose-dependent. ${ }^{11,12}$

However, alternative models of therapies for the treatment of this disease have been promising, one of them being cryotherapy, already used in the treatment of other rheumatic diseases, due to its effects of analgesia, vasoconstriction, muscle relaxation, anti-inflammatory and antioxidative. Still, cryotherapy has been shown to be effective, low cost and easy to use in cases of rheumatoid arthritis and muscle injuries. ${ }^{13-15}$

Therefore, studies are needed to verify the effectiveness of efficient non-pharmacological methods, such as cryotherapy in gouty arthritis, the object of this review, which aims to fill the gaps and point out new efficient treatments, minimizing the effects caused by the disease and for the development of new protocols to promote and maintain the quality of life of the population affected by gouty arthritis. The hypothesis of this study was the existence of research on cryotherapy in individuals with gout, thus, the aim of this study was to gather data on the treatment of cryotherapy in the control of pain in patients with gout, as well as to evaluate the methodological quality of published works.

\section{METHOD}

\section{Search strategy}

This review was registered in the PROSPERO database (CRD42020158265) and was described according to the items requested by the PRISMA system. ${ }^{16}$ This is a systematic review of publications from the year 2000 to the year 2019 in the following white literature databases PubMed, LILACS, SciELO, Web of Science, Science Direct, Scopus, Cochrane, PEDro and in the gray literature a Google Scholar database. The electronic databases were consulted from November 2019.

Three researchers (AT, IR and LP) performed the electronic search independently using a three-component combination strategy: (1) gout AND (2) cryotherapy AND (3) pain. The following terms associated with the booleans were used:

Gout terms: Gout OR gota OR "artrite gotosa" OR "arthritis, gouty" OR “artritis gotosa” OR hyperuricemia
OR hyperuricemia OR "ácido úrico" OR "uric acid” OR artrite OR arthritis OR articulações OR joints OR articulación OR artropatias OR “joint diseases” OR arthralgia AND

Ice terms: crioterapia $\mathrm{OR}$ cryotherapy $\mathrm{OR}$ cryotherapies OR gelo OR ice OR hielo OR frio OR "cold temperature" OR hielo OR "hipotermia induzida" OR "cold therapies" OR imersão OR immersion OR "inmersión en agua fría" OR fisioterapia OR "physical therapy modalities" OR "modalidades terapéuticas" OR "therapeutic use" AND

Pain terms: "acute pain" OR "pain menagement" OR "dolor agudo" OR inflamação OR inflammation OR inflamación.

\section{Inclusion and exclusion criteria}

\section{1) Study design}

The research was restricted to randomized and quasirandomized clinical trials, available in English, Portuguese and Spanish that addressed the treatment of gout using cryotherapy. Non-randomized studies, case reports, clinical observation, book chapters and literature reviews were disregarded. After removing duplicates, titles and abstracts were examined, discarding inaccessible or unclear eligibility articles.

\section{2) Population}

Studies with a sample composed of human beings, adults, with gouty arthritis were considered, and studies that contemplated a sample with other joint injuries and absence of medical diagnosis of the disease were disregarded.

\section{3) Intervention}

Studies that used cryotherapy in joints affected by gout through the application method with an ice or gel bag, immersion in water or use of electronic cooling equipment, with description of the protocol used by a health professional or by patient under guidance.

The presence of other physical therapy or medication interventions did not influence the study's eligibility, but when present, they were considered by the reviewer regarding the influence of their effect.

\section{4) Clinical results}

Studies should present at least one method of nociceptive assessment or functional disability in order to be included in this review.

\section{5) Data extraction}

The following data were obtained: (1) Study type; (2) Sample; (3) Assessment methods; (4) Characteristics of the intervention: type, time and frequency; (5) Main results - 
symptomatic outcomes.

\section{6) Quality assessment}

The articles included in the review had their methodology independently assessed by each researcher. The PEDro scale was used, which classifies the quality of the study as high, regular or poor, based on 11 criteria that resulted in a final score. ${ }^{17}$

\section{RESULTS}

After the initial search, 670 articles were found with titles that presented the selected descriptors, however, after reading the titles and abstracts, 662 were excluded, which did not meet the inclusion criteria, leaving only 9 articles, of which 2 were duplicates and 1 was written in Cyrillic alphabet, then 6 documents were read in full. Then, 2 articles from clinical trials were used for this work, since the other 4 were reviews, according to the diagram shown in Figure 1.

After detailed analysis, two clinical trials were potentially selected, including the methodological criteria stipulated for the intended result (Table 1).

The methodological quality of the selected articles was assessed using the PEDro scale, obtaining a score of 7 for the article by Schlesinger et al. ${ }^{19}$ and 6 for Schlesinger $^{20}$ (Table 2).

\section{DISCUSSION}

Cryotherapy is a physiotherapeutic practice present in several studies that aim to control pain, however the literature is weak and outdated as regards the treatment of cooling for the control of gouty arthritis symptoms. ${ }^{18}$ After the systematic search and categorization of the studies, it can be observed that, even if the resulting post-treatment results are positive, it has a little explored field and with low

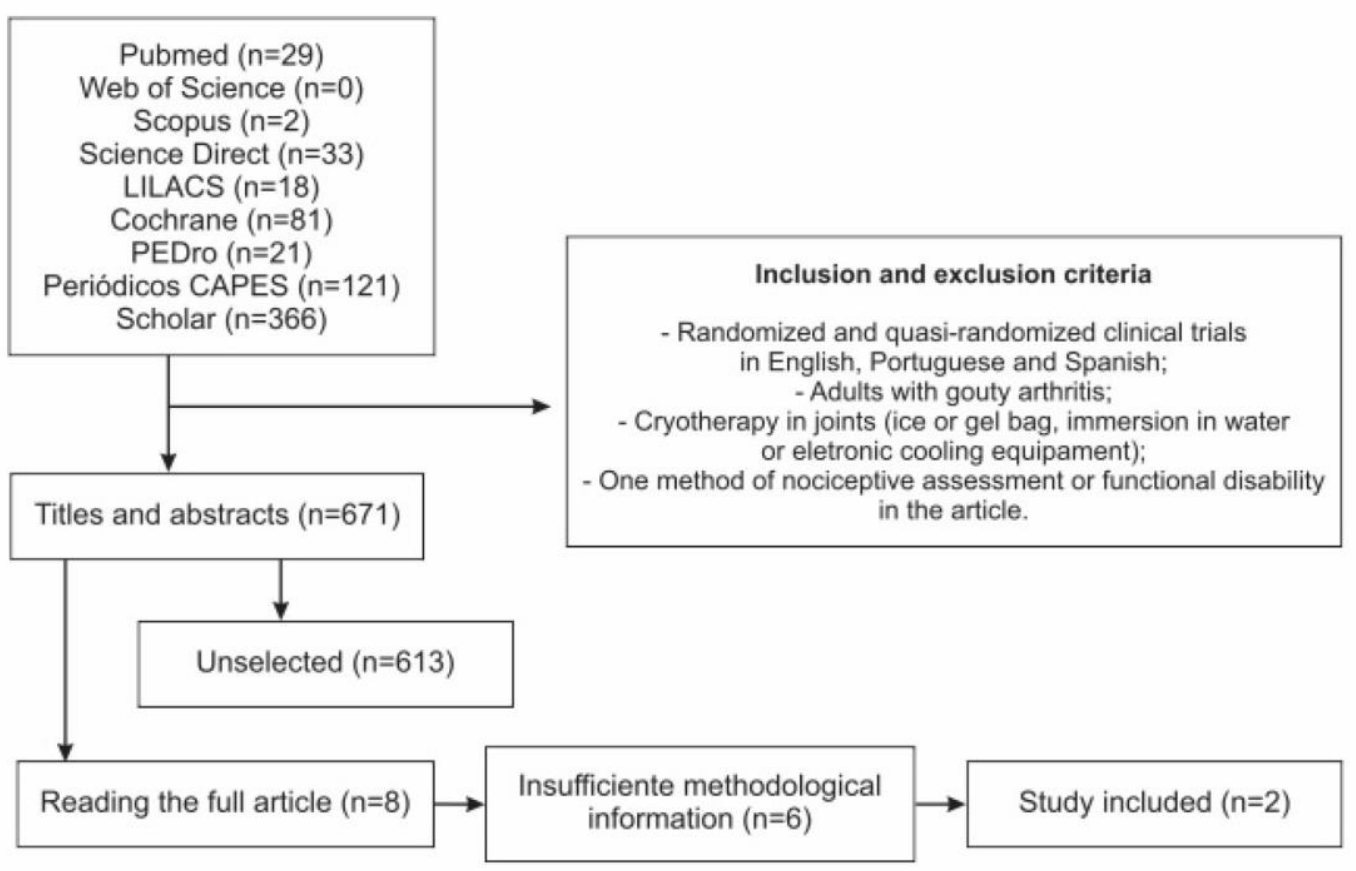

Figure 1. Flowchart of article selection.

Table 1. General data on included clinical trials

\begin{tabular}{cccccc}
\hline Author & Study type & Sample & Evaluation & Intervention & Main results \\
\hline Schlesinger et al. ${ }^{19}$ & $\begin{array}{c}\text { Randomized } \\
\text { clinical trial }\end{array}$ & 19 patients & $\begin{array}{c}\text { Visual analog scale of pain } \\
\text { Joint circumference }\end{array}$ & $\begin{array}{c}\text { Ice pack for 30 minutes, } 4 \\
\text { times a day, for 1 week }\end{array}$ & Pain reduction \\
Schlesinger ${ }^{20}$ & Clinical trial & 20 patients & $\begin{array}{c}\text { Subjective pain } \\
\text { questionnaire }\end{array}$ & $\begin{array}{c}\text { Bag with gel ice for 19 } \\
\text { minutes at intervals of 5 to } \\
\text { 60 minutes }\end{array}$ & $\begin{array}{l}\text { Joint pain } \\
\text { reduction }\end{array}$ \\
\hline
\end{tabular}


Table 2. Stratification of clinical trials using the PEDro scale evaluation

\begin{tabular}{ccc}
\hline Author & Study location & PEDro scale \\
\hline Schlesinger et al. ${ }^{19}$ & Philadelphia & $7 / 11$ \\
Schlesinger $^{20}$ & New Brunswick & $6 / 11$ \\
\hline
\end{tabular}

scientific evidence. Thus, it becomes difficult to develop effective frequency protocols, methods and duration to promote analgesia during the gout pain period.

Consequently, after searching the aforementioned databases, only 2 studies were found, clinical trials, which fit the chosen criteria of this research, both of which are of the same authorship and, carried out for more than ten years, which are Schlesinger author. Due to the scarcity of studies corresponding to the proposed objective, there was a limitation of the possibility of performing a meta-analysis of these since their data are unique. However, its results are relevant and have therefore been considered.

The clinical trial conducted by Schlesinger et al., ${ }^{19}$ aimed to verify the effect of the local application of ice on the duration and severity of the gouty arthritis crisis, using a sample of 19 volunteers, randomized in two groups, in which the experimental group received topical gel applications for 30 minutes, 4 times a day, as an adjunct to drug treatment with oral corticosteroids (Predinisone $30 \mathrm{mg}$ to $0 \mathrm{mg}$ in weaning scheme, with a dose reduction of $10 \mathrm{mg}$ every 2 days) and Colchicine $0.6 \mathrm{mg}$ daily. The control group received only the medications, in the same doses and on the same schedule. The results indicate the report of pain improvement, according to the numerical scale in centimeters, of the experimental group, in $7.75 \mathrm{~cm}( \pm \mathrm{SD} 2.58)$ and 4.42 $\mathrm{cm}( \pm \mathrm{SD} 2.96)$ for the control, with $p=0.021$. However, there was no significance regarding joint circumference, synovial lavage inflammatory cells and volumetric amount of synovial fluid.

Still Schlesinger ${ }^{20}$ carried out another clinical trial, with a sample of 150 volunteers, divided into 6 groups, being patients diagnosed with gout, rheumatoid arthritis, osteoarthritis, other type of inflammatory arthritis or soft tissue pathology. In this experiment, the volunteers answered a questionnaire regarding their perceptions about local pain treatments by means of heat or cold. The authors report that patients diagnosed with gout were the only ones to prefer cold to heat $(p=0.01$ ), however, 3 volunteers who were not carriers of gout but preferred cryotherapy, in an analytical deepening of the results, presented crystals intra-articular urate or inflammation of synovial fluid. Thus, the authors suggest the use of cryotherapy as a diagnostic method. During the period of collection and analysis in the databases, three review articles were observed, but disregarded since all cited the author's work already included. These addressed methods of treatment, management and overview of gouty arthritis, considering cryotherapy as an effective therapy for symptom reduction. ${ }^{21-23}$

Both included researches presented methodological weaknesses, such as the lack of identification of the evaluated joint, of functional and more robust methods for assessing hypernociception without the use of subjective scales. The study Schlesinger et al., ${ }^{19}$ also showed a low n sample, which in turn, directly interferes with the quality of statistical analysis. The two articles also did not describe the way in which the sample was allocated, making it ideal for participants and evaluators to be blinded.

On the PEDro Quality Scale, the article from 2002 and 2006 reached $63 \%$ and $54 \%$ of the total score, respectively. Although both researches corroborate a positive result regarding treatment with cryotherapy, they originate from studies with reasonable methodological quality, confirming the need for new investigations that seek current concepts through solid and rigorous methodologies.

\section{CONCLUSIONS}

Through the search for scientific evidence, it can be concluded that the findings about the effects of cryotherapy in the treatment of pain in individuals with gouty arthritis denote a positive effect, however, there is little evidence to support this hypothesis.

\section{Key Points}

Question Is cryotherapy effective in reducing the pain of individuals with gouty arthritis?

Findings After the initial search, 670 articles were, however, after reading the titles and abstracts, 662 were excluded, which did not meet the inclusion criteria, leaving only 9 articles, of which 2 were duplicates and 1 was written in Cyrillic alphabet, then 6 documents were read in full, and 2 articles from clinical trials were used.

Meaning The treatment of pain in individuals with gouty arthritis denote a positive effect of the cryotherapy, however, there is little evidence to support this hypothesis and the existing ones present reasonable methodological rigor.

\section{Article information}

Conflict of Interest Disclosures: None.

Funding/Support: None.

Acknowledgment: None.

Ethic Approval: None. 


\section{REFERENCES}

1. Mitroulis I, Kambas K, Ritis K. Neutrophils, IL-1 $\beta$, and gout: Is there a link? Semin Immunopathol. 2013;35(4): 501-512.

2. Roddy E, Mallen CD, Doherty M. Gout. BMJ. 2013; 347(7927): f5648.

3. Stamp LK, Jordan S. The challenges of gout management in the elderly. Drugs Aging. 2011;28(8):591-603.

4. Azevedo VF, Lopes MP, Catholino NM, Paiva E dos S, Araújo VA, Pinheiro G da RC. Revisão crítica do tratamento medicamentoso da gota no Brasil. Rev Bras Reumatol. 2017;57(4):346-355.

5. Narang RK, Topless R, Cadzow M, et al. Interactions between serum urate-associated genetic variants and sex on gout risk: analysis of the UK Biobank. Arthritis Res Ther. 2019;21(1):13.

6. Choi HK, Mount DB, Reginato AM. Pathogenesis of gout. Ann Intern Med. 2005;143(7):499-516.

7. Perez-Ruiz F, Marimon E, Chinchilla SP. Hyperuricaemia with deposition: latest evidence and therapeutic approach. Ther Adv Musculoskelet Dis. 2015;7(6):225-233.

8. Dalbeth N, Merriman TR, Stamp LK. Gout. Lancet. 2016; 388(10055):2039-2052.

9. Desai J, Steiger S, Anders HJ. Molecular pathophysiology of gout. Trends Mol Med. 2017;23(8):756-768.

10. Finch A, Kubler P. The management of gout. Aust Prescr. 2016;39(4):119-141.

11. Assis L, Milares LP, Almeida T, et al. Aerobic exercise training and low-level laser therapy modulate inflammatory response and degenerative process in an experimental model of knee osteoarthritis in rats. Osteoarthr Cartil. 2016;24(1):169-177.

12. Khanna D, Fitzgerald JD, Khanna PP, et al. 2012 American college of rheumatology guidelines for management of gout. part 1: systematic nonpharmacologic and pharmacologic therapeutic approaches to hyperuricemia. Arthritis Care Res. 2012;64(10):1431-1446.

13. Martins CN, Moraes MB, Hauck M, et al. Effects of cryotherapy combined with therapeutic ultrasound on oxidative stress and tissue damage after musculoskeletal contusion in rats. Physiother (United Kingdom). 2016; 102(4):377-383.

14. Guillot X, Tordi N, Mourot L, et al. Cryotherapy in inflammatory rheumatic diseases: a systematic review. Expert Rev Clin Immunol. 2014;10(2):281-294.

15. Peres D, Sagawa Y, Dugué B, Domenech SC, Tordi N, Prati C. The practice of physical activity and cryotherapy in rheumatoid arthritis: systematic review. Eur J Phys Rehabil Med. 2017;53(5):775-787.

16. Liberati A, Altman DG, Tetzlaff J, et al. The PRISMA statement for reporting systematic reviews and metaanalyses of studies that evaluate health care interventions: explanation and elaboration. J Clin Epidemiol. 2009;62(10):e1-e34.

17. PEDro Evidence. Escala de PEDro - Português (Portugal) 1. Pedro Phisicotherapy Evid Base. Published online 2009:1-7.

18. Gutiérrez Espinoza HJ, Lavado Bustamante IP, Méndez Pérez SJ. Systematic review of the analgesic effect of cryotherapy in the management of musculoskeletal pain. Rev la Soc Esp del Dolor. 2010;17(5):242-252.

19. Schlesinger N, Detry MA, Holland BK, et al. Local ice therapy during bouts of acute gouty arthritis. J Rheumatol. 2002;29(2):331-334.

20. Schlesinger N. Response to application of ice may help differentiate between gouty arthritis and other inflammatory arthritides. J Clin Rheumatol. 2006;12(6):275276.

21. Cheng L, Che Y, Am G. Lifestyle interventions for acute gout (Review). Cochrane Database Syst Rev. 2013;(8): 1-25.

22. Khanna PP, Gladue HS, Singh MK, et al. Treatment of acute gout: a systematic review. Semin Arthritis Rheum. 2014;44(1):31-38.

23. Moi JHY, Sriranganathan MK, Falzon L, Edwards CJ, Van Der Heijde DM, Buchbinder R. Lifestyle interventions for the treatment of gout: a summary of 2 cochrane systematic reviews. J Rheumatol. 2014;41(SUPPL. 92): 26-32. 